\title{
Investigation of the relationship between the Lamb waves phase velocity and the technical condition of housing and utilities pipelines
}

\author{
Ibadov A.A. ${ }^{1, *}$, Kondrat'ev A.E. ${ }^{1}$, Makueva D.A. ${ }^{1}$ and Sergeeva D.V. ${ }^{1}$ \\ ${ }^{1}$ Kazan State Power Engineering University, Kazan, Russian Federation
}

\begin{abstract}
The article describes a method for determining the dependence of the Lamb waves phase velocity on the technical condition of housing and utilities pipelines, using the example of thin two-layer segments. Variations in the thickness of the considered pipeline affect the propagation parameters of the Lamb wave mode.
\end{abstract}

\section{Introduction}

During long-term operation, pipelines are exposed to internal and external influences. Because of them, material destruction occurs, corrosion appears and other types of defects develop. The use of modern materials and technologies for the manufacture and laying of pipelines does not exclude the possibility of various deformations [1].

It is very effective to use metallic and non-metallic coatings to ensure the wear resistance and durability of steel objects of thermal power plants. But this requires determining and evaluating the quality and area of adhesion of materials to the base.

Monitoring by non-destructive testing methods remains relevant to solve such problems. This helps to reduce the occurrence of undesirable consequences from the degradation of pipeline materials developing over time. Traditional non-destructive testing methods have several disadvantages. They are associated with the limitation of the application scope to a certain set of materials, and also have a weak selectivity in monitoring the location of pipelines [2]. It is proposed to consider a new approach to determine the technical condition of pipelines for housing and utilities. It is based on the propagation of Lamb waves in the considered segments.

\section{Lamb waves propagation in a segment}

Consider a segment with a thickness of $2 d$, in which the origin is taken as $Y$. In this case, the wave will propagate along the $X$ axis, and the normal will be directed along the $Z$ axis (Fig.1).

We begin our investigation of the pipelines vibroacoustic parameters with the equation below. It describes the propagation of elastic waves in segments homogeneous in structure through the scalar $\varphi_{l}$ and the vector potential $\psi_{l}$, which, according to [3], has the form:

$$
\begin{aligned}
& \Delta \varphi_{l}=k_{l}^{2} \varphi_{l}=0 \\
& \Delta \psi_{l}=k_{t}^{2} \psi_{l}=0
\end{aligned}
$$

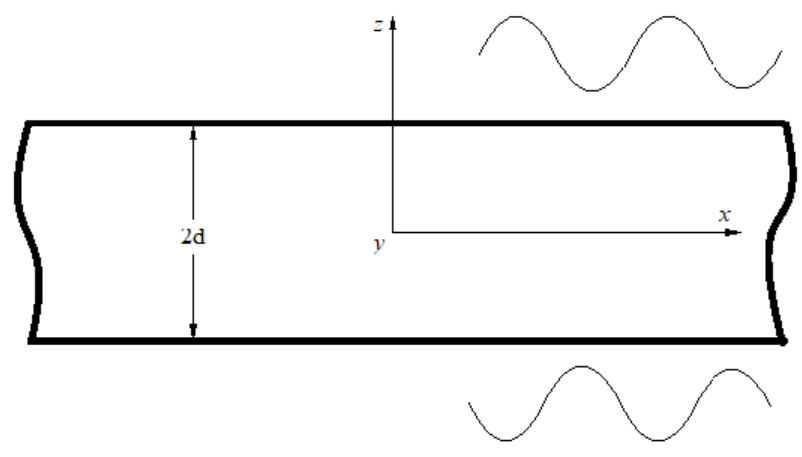

Fig. 1 - problem geometry

The wave numbers of longitudinal and transverse waves are equal, respectively

$$
\begin{aligned}
& k_{l}=2 \pi v\left[\rho /\left(\lambda_{l}+2 \mu\right)\right]^{1 / 2} \\
& k_{t}=2 \pi f(\rho / \mu)^{1 / 2}
\end{aligned}
$$

where $\rho$ is the density of the medium; $\lambda_{l}$ and $2 \mu$ are elastic constants.

Acoustic waves are characterized by geometric dispersion in contrast to surface waves. Since $d$ is constant over the entire length of the segment, and the wavelength is $\lambda=2 \pi C / \omega=2 \pi / k$, it becomes obvious that the ratio between the wavelength and the segment thickness depends on $\omega$. Here $\omega-$ is the cyclic frequency, and $C-$ is the acoustic wave speed. Therefore, there is dispersion for all waves in the segment [4]. The connection of scalar and vector

* Corresponding author: amil@mail.ru 
potentials with displacement components along the $X$ and $Z$ axes are given in [3]. The stress tensor components $\sigma_{i j}$ on the segment planes $z= \pm d / 2$ should tend to zero. Based on this, we transform the formulas (1) and (2):

$$
\begin{aligned}
& \varphi=A_{S} \operatorname{ch} q z e^{i k x}+B_{A} s h q z e^{i k x} \\
& \psi=D_{S} s h s z e^{i k x}+C_{A} c h s z e^{i k x}
\end{aligned}
$$

where $q=\left(k^{2}-k_{l}^{2}\right)^{1 / 2} ; s=\left(k^{2}-k_{t}^{2}\right)^{1 / 2} ; A_{S}, B_{A}, C_{A}, D_{S}$ are the arbitrary constants; $k$ - the Lamb wave number. In this case, the multiplier $e^{-i \omega t}$ can be neglected in the calculations.

Based on the equations obtained in [3], we note that these groups of waves describe the asymmetric and symmetric motion of waves in the plane $Z=0$.

Analysis of asymmetric and symmetric waves in the segment shows that they are characterized by a strong speed dispersion. The waves propagation speed $C_{L}$ depends on the segment thickness, the elastic properties of the material and the wave frequency $\omega$. From these considerations, we come to the conclusion that for a segment thickness $d$, the propagation speed of the fastest symmetric mode $S_{0}$ can be written as:

$$
C_{S 0}=\left\{E /\left[\rho\left(1-\boldsymbol{N}^{2}\right)\right]\right\}^{1 / 2}
$$

where $E$ is the Young's modulus; $\aleph$ is the Poisson's ratio, which for a wide class of metals is taken in the range from 0.26 to 0.35 .

If we know the dispersion curves of Lamb waves phase velocities, then we can calculate the displacements and stresses in them [3]. The longitudinal $U_{S O}$ and transverse $W_{S 0}$ displacements of the wave on the segment are as follows:

$$
\begin{gathered}
U_{S O}=A k_{t}\left[\left(k_{S}^{2}-S_{S}^{2}\right) \sin \left(k_{S} x-\omega t\right)\right] /\left[k_{t} d\left(k_{S}^{2}+S_{S}^{2}\right)\right] \\
W_{S 0}=U_{S O} q_{s} z \operatorname{ctg}\left(k_{S} x-\omega t\right)
\end{gathered}
$$

In accordance with the given equations, longitudinal waves are dominated by displacements along the $X$ axis with the same amplitude for all points of the segment. The displacement in the transverse direction is much less than the longitudinal displacement due to the Poisson effect [5].

\section{Theoretical analysis}

Let us consider the situation of wave propagation in a two-layer pipe, in which both layers are made of different materials. We accept the total thickness of the base and coating less than $1 \mathrm{~mm}$. We will consider a part of the pipe as a thin segment. The base is made of alloy steel with $\boldsymbol{N}=0,28$, and the outer monolayer polyethylene coating has $\boldsymbol{N}=0,42$.

Materials differ in elastic properties from each other, therefore, the following condition is fulfilled:

$$
\left\{\left(k_{t}\right)_{1} d_{1},\left(k_{t}\right)_{2} d_{2}\right\} \ll 1
$$

The main characteristics of the segment materials are shown in the table:

Table 1. Main characteristics of materials

\begin{tabular}{|c|c|c|c|c|c|c|c|}
\hline & $E$ & $N$ & $\rho$ & \multicolumn{3}{|c|}{$d$} \\
\hline & $M P a$ & & $k g / m^{3}$ & \multicolumn{3}{|c|}{$\mu m$} \\
\hline $\begin{array}{c}\text { Monolayer } \\
\text { polyethylene } \\
\text { coating }\end{array}$ & 770 & 0.42 & $9,65 \times 10^{2}$ & 1 & 2 & 3 & 4 \\
\hline Alloy steel & $2,2 \times 10^{5}$ & 0.28 & $7,85 \times 10^{4}$ & 22 & 36 & 45 & 51 \\
\hline
\end{tabular}

If condition (10) is satisfied and there is a defect, for example, there is no adhesion between the layers, then the $S_{0}$-mode will propagate in the steel base of the segment and in the coating separately. Its speed $\left(C_{S 0}\right)_{1}$ and $\left(C_{S 0}\right)_{2}$, will be determined by formula (7), for the main and outer layers, respectively.

We also assume that the rigid adhesion condition of the contact surfaces of the segment materials is fulfilled:

$$
\begin{aligned}
& \left(\sigma_{i j}\right)_{1}=\left(\sigma_{i j}\right)_{2} \text { and }\left(U_{S 0}\right)_{1}=\left(U_{S 0}\right)_{2} ; \\
& \left(W_{S 0}\right)_{1}=\left(W_{S 0}\right)_{2} \text { when } z=0
\end{aligned}
$$

Then we summarize the parameters for both layers:

$$
\begin{aligned}
& E=1 /\left(E_{1}{ }^{(-1)} d_{1}{ }^{\prime}+E_{2}{ }^{(-1)} d_{2}{ }^{\prime}\right) ; \\
& \boldsymbol{N}=\boldsymbol{N}_{1} d_{1}{ }^{\prime}+\boldsymbol{N}_{2} d_{2} ; \rho=\rho_{1} d_{1}{ }^{\prime}+\rho_{2} d_{2}{ }^{\prime},
\end{aligned}
$$

where $d_{1}{ }^{\prime}=d_{1} / d ; d_{2}{ }^{\prime}=d_{2} / d$.

Since $d_{12}=d_{1} / d_{2}$ is in the range from 0 to 1 , then formula (7) will look like:

$$
C_{S 0} \approx\left\{1-\left[d_{1}\left(E_{21}+\rho_{12}-2\right)\right] / 2\right\}\left(1+\boldsymbol{N}_{2}^{2} / 2\right)\left(E_{2} / \rho_{2}\right)^{1 / 2}
$$

Thus, at $\rho_{2} E_{2} \neq \rho_{1} E_{l}$, a change in the segment thickness is observed [5].

For the numerical calculation of the theoretical analysis, we will solve the problem where we assume that an emitter with an operating frequency is located on the basis of the segment $f=1 \mathrm{MHz}$. Two piezoelectric sensors are located on the surface at an equal distance $L=80 \mathrm{~mm}$, which register the transmitted signal from the emitter. The propagation time of a vibroacoustic signal between two sensors $t=0,1 \mathrm{~s}$ makes it possible to determine the speed of the investigated mode by the formula $C_{S 0}{ }^{*}=L / t_{12}$.

According to the formula (13), $d_{i}$ will be equal to:

$$
d_{1} \approx\left(C_{S 0}{ }^{*} /\left\{\left[1+\left(\boldsymbol{N}_{2}{ }^{2} / 2\right)\right]\left(E_{2} / \rho_{2}\right)^{1 / 2}+1\right\} /\left(E_{21}+\rho_{12}-2\right.\right.
$$

The calculated wave propagation data in a steel segment with a monolayer polyethylene coating is a monotonically decreasing curve, where $\Delta C_{S 0}=C_{S 0}-C_{S 0}{ }^{*}$. The dependence of the propagation speed change of the fastest symmetric mode $S_{0}$ on the segment thickness is shown in Fig. 2. 


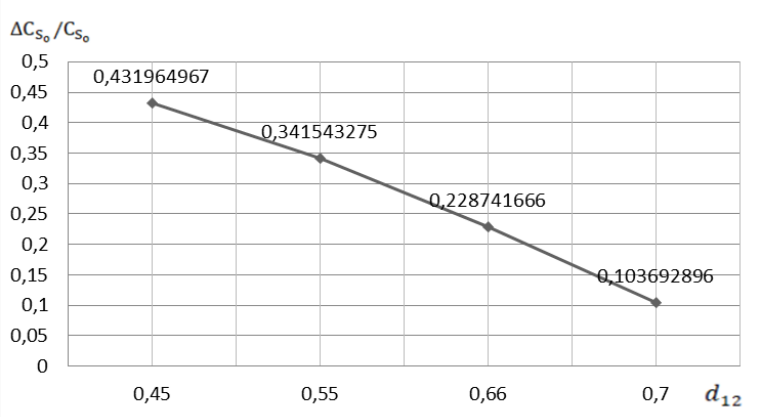

Fig. 2 - Dependence of the wave propagation speed on the segment thickness

\section{Conclusion}

A numerical-analytical method for calculating the Lamb wave mode propagation in a thin two-layer segment is used. The calculation steps are based on the method for determining the propagation speed of the fastest symmetric mode $\mathrm{S}_{0}$. Using the above formulas, the dependence of the wave propagation speed on the segment thickness is shown. Developing this technique, it is possible to record the relative changes in the utility pipelines thickness. This will help to identify changes in the physical properties of the material or a defect presence.

\section{References}

1. Afanasyev V.B., Chernova N.V. Modern methods of non-destructive testing // Successes of modern natural science. No. 7, 73-74 (2011)

2. Gaponenko S.O., Kondratyev A.E. Acoustic method for determining hidden hollow objects of complex shape / International Publishing House "Lambert Academic Publishing" (Germany). 136 (2014)

3. Viktorov I.A., Sound surface waves in solids / I.A. Viktorov. - M .: Nauka, (1981)

4. Zolotova OP, Burkov S.I., Sorokin B.P. Propagation of Lamb and SH-waves in a piezoelectric cubic crystal plate // Journal of Siberian Federal University. Mathematics \& Physics, No. 3 (2), 185204 (2010)

5. Baev A.R., Prokhorenko P.P. Features of propagation of Lamb waves in thin two-layer materials // Bulletin of BNTU. No.4, 52-55 (2008) 